\title{
Issues in Global Software Development: A Critical Review
}

\author{
Sami ul Haq ${ }^{1 *}$, Mushtaq Raza ${ }^{2}$, Asraf Zia ${ }^{2}$, M. Naeem Ahmed Khan ${ }^{1}$ \\ ${ }^{1}$ Shaheed Zulfikar Ali Bhutto Institute of Science and Technology (SZABIST), Islamabad, Pakistan; ${ }^{2}$ Abdul Wali Khan University, \\ Mardan, Pakistan. \\ Email: *uomiansame007@gmail.com
}

Received August $11^{\text {th }}, 2011$; revised September $5^{\text {th }}, 2011$; accepted September $27^{\text {th }}, 2011$.

\begin{abstract}
A number of companies have employed Global Software Development (GSD) methodology as a useful tool for their software development practices. GSD is a contractual relationship between client and vendor organizations in which a client outsources all or some part of its software development activities to a vendor. The vendor in return provides the agreed services in lieu of certain amount of remuneration. The main reasons to select the GSD technique include reduced cost, faster development and access to skilled manpower. Though GSD is emerging as an effective technique, but it suffers from many challenges like poor communication, lack of trust and coordination. These challenges pose serious risk to the smooth execution of the GSD projects. In this paper, we present a comparative study on GSD to highlight its merits and demerits. Our findings reveal that much of the research in this area has been focused on addressing issues faced by client organizations, however, vendor side in the GSD relationship is much ignored due to which this area is still immature; and, hence, further research work is required to be undertaken to address the issues faced by the vendor organizations.
\end{abstract}

Keywords: Communication, Co-Ordination and Trust, Global Software Development (GSD), Distributed Software Development (DSD)

\section{Introduction}

Global Software Development, also known as Distributed Software Development, is originally an outsourcing technique in which the development teams hailing from diverse cultural backgrounds and located at distant remote locations participate in the software development efforts and provide their services through a tightly-coupled computer networks like Internet. The sole reason for choosing this technique is due to its cost effectiveness as outsourcing organizations usually outsource their contracts to the country where the labor cost is comparatively low). Despite the fact that GSD is widely being used, however, the project managers dealing the GSD projects face many challenges in planning, managing and execution of such types of projects. The major challenges include communication, coordination and trust with the key stakeholders. Besides, cost and time estimation for GSD are also the prime issue that GSD project managers occasionally face. A lot of work has been carried out to address these issues like Sangwan and Ros [1] have highlighted that the role of architect and Korkala et al. [2] stressed that customers should never be ignored. These issues have resulted in emergence of agile methodology for GSD. The communication and coordination among developers and testers matters a lot [3]. Though Rammasubbu and Balan [4] have presented research directions in GSD project planning, execution and reflection phases, but an ideal solution to these problems still lacks.

This paper is organized into six sections. The next section provided a brief background of the GSD problems. Literature review is summarized in the third section followed by critical review of the major GSD techniques in the preceding section. The fifth section discusses the prospective future work and finally we conclude in the last section.

\section{Background}

Outsourcing is a state of affairs in which a company imports all or some parts of their product from another vendor [5]. Outsourcing has different subtypes e.g., onshore or domestic outsourcing (in which both clients and vendors are from same country but different locations), near-shore outsourcing (in which the teams are from 
neighbor countries) and Off-shore outsourcing (in which teams are from the far-flung regions/countries) [5].

Global Software Development, Distributed Software Development and outsourcing are primarily different names for a unique software development technique through which the clients get services from an outside organization known as vendors. This technique is of the interest to the clients due to lower labor cost, faster delivery and access to skilled human resources. Despite the supplementary benefits, this technique suffers from some limitations like: teams involved in the software development activities usually have different cultural backgrounds; face to face meetings are difficult due to their remote locations; time zones and working hours of the clients and vendors do not match; there are language barriers among both the parties which lead to communication and coordination issues; and trust between both the parties always remain a major concern.

\section{Literature Review}

A number of researchers have tried to address different issues of GSD. A detailed account of the issues/problems being faced by GSD and their solutions proposed by different researchers is provided in this section.

Sangwan and Ros [1] stress that the communication, coordination and control mechanisms are major issues in GSD. Therefore, the role of an architect is very important to share the common context of the system for all the involved teams in GSD. The research emphasizes on adaptation of Architectural Description Languages (ADL), but this approach suffers a major limitation as conflict among teams may occur in the understanding of ADL due to availability of a number of ADLs.

Korkala et al. [2] applied traditional and agile methods in GSD. The findings of the study show that agile methods provide better results in GSD. However, the study suffers from the limitation that the data was collected from only one group and other teams were ignored. Agile methods have their own limitations like due to too many meetings with the customers, they may lose their interest. This technique is beneficial in large projects where rapid application is required. But in case of small projects, the project managers may not feel comfortable to maintain a team on customer site for communication and coordination due to budget constraints. The framework for the communication channels proposed by Korkala et al. [2] is shown in Figure 1.

Grechanik et al. [3] discussed communication and coordination challenges among developers and testers and their impacts on the overall project. The research identifies many issues that can arise due to poor communication and coordination between testers and developers as well as highlighting their impact on project. However, a fitting framework or model is required for the communication and coordination between developers and testers to resolve these issues.

Rammasubbu and Balan [4] point out certain research directions to enhance the governance scheme for Distributed Software Development projects. The findings include the identification of major issues in project planning, execution and reflection phases.

Khan et al. [5] identify various barriers that can have negative impacts on the software outsourcing clients in the selection process of offshore software development outsourcing vendors. The study identifies sixteen such factors including communication gap, country instability, delays in delivery, hidden costs, incompatibility with client, lack of Project Management practices, lack of protection for intellectual property rights, lack of technical capability, language and cultural barriers, lack of control over project, poor quality of service and system/process, opportunistic behavior, poor contract management, poor infrastructure, poor relationship management and strategic inflexibility. The study does not, however, throw light how to tackle or avoid these barriers.

Jalote and Gupta [6] assert that the use of global pairs can reduce the development time provided that the global pairs are created by following the sun phenomenon. The assertion is mainly made on the basis of work nature of the developer and tester. The global pairs can reduce software development time especially when developers and testers are from different time zones. However, the technique is applicable in the development and testing areas. Other phases of overall software development activities also need to be examined in the context of global pairs.

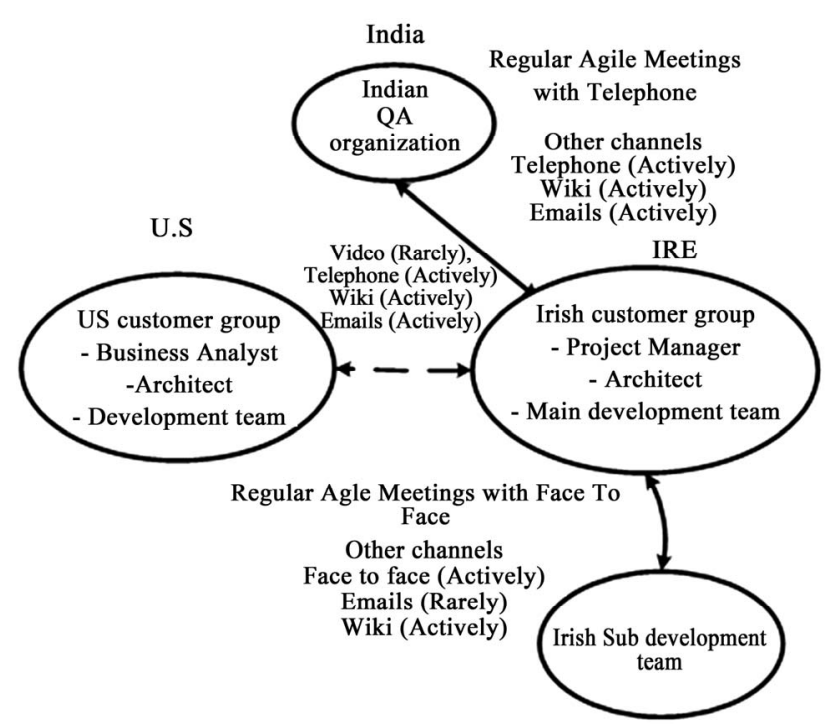

Figure 1. The communication channels and their usage in the case projects [2]. 
Ju [7] describes the efforts made by China to secure a market place in software development in general and IT industry in particular through its unique strategies and policies.

Casey [8] emphasizes the importance of imparting GSD education to students and stresses the importance of bringing about the GSD culture. The study highlights that there is a lack of awareness about GSD among the IT students. The framework for impact of distance in GSD environment as proposed by Casey [8] is exhibited in Figure 2.

Nguyen et al. [9] state that the establishment and maintenance of trust is a major issue in GSD. The study is based upon the analysis of data collected from eight companies. For establishing trust, most of the companies recommend to bring about cultural understanding, creditability, capabilities, pilot project performance, personal visits and investments in the field of GSD. The study also suggests cultural understanding, capabilities, contract conformance, quality, timely delivery, development processes, managing expectations, personal relationships and performance as the key factors for maintenance of trust in communication,. The study needs further investigation and to enlarge its canvas as the current data is collected only from a single location.

Wiredu [10] present a framework for the analysis of coordination in GSD. The framework provides good understanding of the analysis of coordination issues in GSD, but the framework needs experimental validation in the real environment.

Carmel and Abbott [11] perform a systematic literature review to find out to what extent the distances matter in GSD efforts. The study also provides detailed information about near-shoring concept. The study also focuses on the advantages of near-shoring over far-shoring. The study reveals that the distances between the client and vendor locations do matter a lot.

Ramingwong and Ramingwong [12] discuss advantages and disadvantages of outsourcing in terms of cost, unemployment, quality, skills, 24/7 working operations, culture and language. The study is much informative to understand the advantages and disadvantages of GSD in the global scenario. A comparative analysis of benefits and risks associated with GSD with respect to different parameters as identified by Ramingwong and Ramingwong [12] is replicated in Figure 3.

Hazzan and Dubinsky [13] claim that diversity in distributed development system can be enhanced by employing agile software development methodology. The study discusses three diversity issues namely gender diversity, management diversity and opinion diversity from Agile software development perspective. The study stresses that the role chart is a useful technique for man- agement diversity. The study, however, does not address other diversity issues in GSD like cultural diversity, language diversity, time zone diversity etc.

Conchuir et al. [14] use a survey based approach to identify the benefits of GSD. The reports six main influencing benefits of GSD which are 1) reduced development cost; 2) time zone effectiveness; 3) cross site modularization of work; 4) access to large skilled labor pool; 5) innovation and shared best practices; and 6) closer proximity to market and customer. However, the study does not account for the implementation methodology to address/implement these factors.

Setamanit et al. [15] propose a simulation model which on the whole is an automated tool that assists the project managers. By using this tool, the project manager can monitor the project from different dimensions and parameters. It also facilitates the project managers in taking critical decisions e.g., relating different sites to different tasks and evaluating their suitability. The model can easily identify both positive and negative aspects of each site as well asserting the specialty of each site. However, to check the performance, efficiency and productivity of the tool, it needs to be applied in large GSD projects.

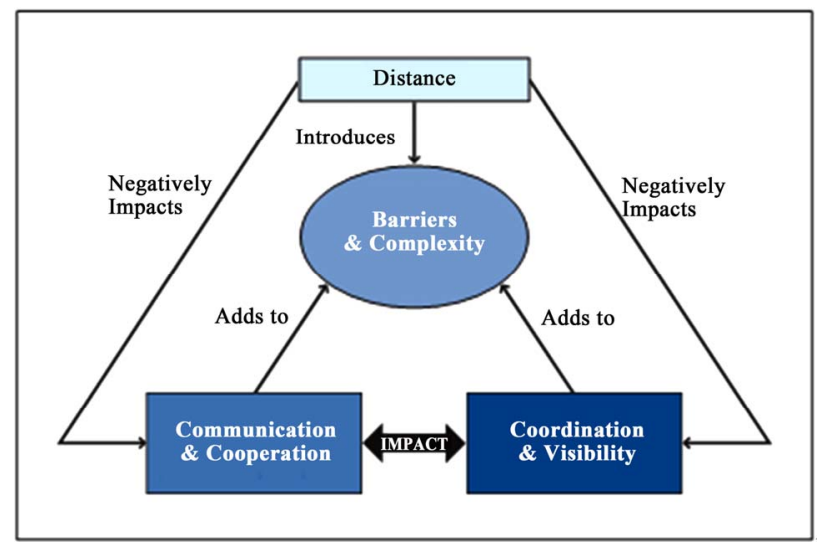

Figure 2. The impact of distance in GSD environment [8].

\begin{tabular}{|c|c|c|}
\hline & Benefits & Risks \\
\hline Costs & Low labour costs & $\begin{array}{c}\text { Additional and hidden } \\
\text { Costs }\end{array}$ \\
\hline Unemployment & $\begin{array}{c}\text { Increasing needs of } \\
\text { global talents }\end{array}$ & $\begin{array}{c}\text { Unemployment in major } \\
\text { nations }\end{array}$ \\
\hline Skills & $\begin{array}{c}\text { Infinite pool of } \\
\text { workforce }\end{array}$ & $\begin{array}{c}\text { Insufficient level of } \\
\text { required skills }\end{array}$ \\
\hline 24/7 Operation & $\begin{array}{c}\text { Around-the-clock } \\
\text { operation }\end{array}$ & $\begin{array}{c}\text { Lack of instantaneous } \\
\text { communication }\end{array}$ \\
\hline Quality & $\begin{array}{c}\text { Possibility of } \\
\text { Satisfactory products }\end{array}$ & $\begin{array}{c}\text { Possibility of inadequate } \\
\text { differences }\end{array}$ \\
\hline Culture & $\begin{array}{c}\text { Broader perspectives } \\
\text { and opportunities }\end{array}$ & $\begin{array}{c}\text { Problems from cultural } \\
\text { differences }\end{array}$ \\
\hline Language & $\begin{array}{c}\text { Encouraging } \\
\text { localization }\end{array}$ & $\begin{array}{c}\text { Problems from language } \\
\text { barriers }\end{array}$ \\
\hline
\end{tabular}

Figure 3. Benefits and risks of GSD [12]. 
Table 1. Critical analysis of GSD literature.

\begin{tabular}{|c|c|c|}
\hline Ref\# & Research Theme & Key Features \\
\hline [1] & $\begin{array}{l}\text { Good quality architectural leadership } \\
\text { and management are required in GSD. }\end{array}$ & The role of architect is highlighted \\
\hline [2] & $\begin{array}{l}\text { Blending traditional and agile } \\
\text { approaches for GSD. }\end{array}$ & $\begin{array}{l}\text { The combination of agile and traditional approaches is } \\
\text { beneficial for GSD. }\end{array}$ \\
\hline [3] & $\begin{array}{l}\text { Gauge the efficacy of communication } \\
\text { and coordination among testers and } \\
\text { developers in GSD environment. }\end{array}$ & $\begin{array}{l}\text { Highlights issues that arise due to poor communication } \\
\text { and coordination among testers and developers; and } \\
\text { determines their negative impacts on the project. }\end{array}$ \\
\hline [4] & $\begin{array}{l}\text { Discussion on research directions in } \\
\text { governance scheme for Distributed } \\
\text { Software Development projects. }\end{array}$ & $\begin{array}{l}\text { Issues related to project planning, execution and } \\
\text { reflection phases for DSD projects are identified. }\end{array}$ \\
\hline [5] & $\begin{array}{l}\text { Identification of barriers in the } \\
\text { selection of offshore vendors. }\end{array}$ & $\begin{array}{l}\text { Study spotlights sixteen factors that act as barriers in the } \\
\text { selection of an offshore vendor. These factors are: } \\
\text { communication gap, country instability, delays in } \\
\text { delivery, hidden costs, incompatibility with client, lack } \\
\text { of Project Management practices, lack of protection for } \\
\text { intellectual property rights, lack of technical capability, } \\
\text { language and cultural barriers, lack of control over } \\
\text { project, poor quality of service and system/process, } \\
\text { opportunistic behavior, poor contract management, poor } \\
\text { infrastructure, poor relationship management and } \\
\text { strategic inflexibility. }\end{array}$ \\
\hline
\end{tabular}

[6] Usage of global pairs in GSD

[7] Outline guidelines and policies for capturing IT and software market.

[8] Importance of GSD education for the students

[9] How to establish and maintain trust in GSD.

[10] Analysis of coordination in GSD.

[11] A comparative analysis of off-shore vs. near-shore outsourcing.

[12] Merits and demerits of outsourcing.

[13] Enhancement of diversity in GSD through agile software development.

Reduced software development time and cost. Ideally, developers and testers are based on locations with different time zones.

Exploration of efforts made by China towards GSD.

Research shows that there is lack of GSD education in the students and this problem needs to be addressed.

Some parameters for the establishment and maintenance of trust in the GSD environment are proposed.

A framework is provided for better understanding and analysis of coordination issues in GSD.

Study shows that the distances matters a lot in GSD.

Advantages and disadvantages of outsourcing are discussed in terms of cost, unemployment, quality, skills, and 24/7 working operations, culture and language.

The study reveals that agile software development can address the issues of gender, management and opinion diversity in GSD.

[14] Identification of key benefits of GSD. Six success factors in GSD are pointed out which are: reduced development cost; time zone effectiveness; cross site modularization of work; access to large skilled labor pool; innovation and shared best practices; and closer proximity to market and customer.

[15] Need for creation of an automated tool Tool can identify both positive and negative aspects of to assist project managers. each development site for outsourcing purposes, thus helps project managers in making critical decisions.

\section{Key Considerations}

Selection of a ADL language would be a critical decision due to availability of many ADL languages.

Agile methods have their own limitations.

Proper framework is required to be formulated for communication and coordination between tester and developer.

Solution for the issues highlighted in the research needs to be sought.

In addition to development and testing, other phases of software development activities need to be included in the context of global pairs.

Primarily, a marketing approach for IT and software industry.

Parameters are extracted from the data collected from a single location and more data needs to be gathered to determine further parameters.

Framework needs experimentation validation to evaluate its effectiveness.

Other diversity issues in GSD like cultural, language, etc. also need to be explored.

The study does not account for the implementation methodology to address/implement these factors.

Tool needs to be applied in large GSD project to check its performance, efficiency and proclivity. 


\section{Critical Review}

In this section we summarize critical review (Table 1) of the different issues, methodologies, tools and techniques of GSD that have been observed during the course of literature survey of this study. The different aspects of GSD that have been accounted for in this analysis include: architectural management, employing fast software development methodologies, addressing communication issues and maintaining trust in GSD projects. The analysis also accounts for the need for developing and promoting GSD tools to exploit true benefits of outsourcing by following defined set of guidelines and policies that exclusively target the GSD projects.

\section{Future Work}

In this study, we have discovered that most of the researchers have worked on the key issues of GSD that the clients or outsourcing organizations face but hardly anyone has focused on the problems faced by vendors or suppliers while undertaking GSD projects. In this connection, we intend to address this issue in our future research. Our prospective future work will be based on the following two research questions.

RQ.1: What are the challenges faced by the vendors at the planning phase of the GSD projects?

RQ.2: What are the best practices to address the challenges faced by suppliers/vendors in handling the outsource projects?

To find answers for the aforementioned research question, we plan to conduct a systematic literature review to identify the key challenges in this area as well as to discover the best practices being followed within the industry in this regard. We intend to conduct a detailed survey and interviews with the industry practitioners to better understand the current state of the affairs on this issue.

\section{Conclusions}

Outsourcing is becoming more and more popular due to availability of cheap labor costs in certain parts of the world. All the subtypes or paradigms of outsourcing like offshore outsourcing, far-shore outsourcing, offshore software development outsourcing (OSDO), near-shore outsourcing and on-shore outsourcing are widely being used by the global software industry. Though outsourcing is a cost effective technique, but it also suffers from key challenges on both the client and the vendor sides. The issues of communication, coordination and establishment of trust are of prime concerns for both the parties. The differences in time zone at the locations of clients and vendors of the outsourcing projects make the direct communication more difficult which at time leads to serious misunderstanding. Differences in culture and lan- guages of the outsourcing and outsourced country also poses serious challenges for both the vendor and the client. India and China are at the top of the recipient of the offshore western outsourced projects as they have emerged as a champion of the software industry. This study conducted a critical analysis of the existing outsourcing techniques and the key challenges faced by the companies in this regard. The study finds out that there is need for extensive research to be conducted in this area to find solution of the problems faced by the vendors and clients of the outsourced projects to facilitate the core concept of global software development.

\section{REFERENCES}

[1] R. S. Sangwan and J. Ros, “Architectural Leadership and Management in Globally Distributed Software Development," Proceedings of the 1st International Workshop on Leadership and Management in Software Architecture, Leipzig, 10-18 May 2008, pp. 17-22. doi:10.1145/1373307.1373313

[2] M. Korkala, M. Pikkarainen and K. Conboy, “A Case Study of Customer Communication in Globally Distributed Software Product Development," Proceedings of the 11th International Conference on Product Focused Software, Limerick, 21-23 June 2010, pp. 43-46. doi:10.1145/1961258.1961269

[3] M. Grechanik, J. A. Jones, A. Orso and A. van der Hoek, "Bridging Gaps between Developers and Testers in Globally, Distributed Software Development," Proceedings of the FSE/SDP Workshop on Future of Software Engineering Research, Santa Fe, 7-11 November 2010, pp. 149-154. doi:10.1145/1882362.1882394

[4] N. Rammasubbu and R. K. Balan, "Towards Governance Scheme for Distributed Software Development Projects," Proceedings of the 1st International Workshop on Software Development Governance, Leipzig, 10-18 May 2008, pp. 11-14. doi:10.1145/1370720.1370725

[5] S. U. Khan, M. Niazi and R. Ahmad, "Factors Influencing Clients in the Selection of Offshore Software Outsourcing Vendors: An Exploratory Study Using a Systematic Literature Review," The Journal of Systems and Software, Vol. 84, No. 4, 2010, pp. 686-699. doi:10.1016/j.jss.2010.12.010

[6] P. Jalote and A. Gupta, "Using Global Pairs for Reducing Software Development Time," Proceedings of the 4th India Software Engineering Conference, Thiruvananthapuram, 24-27 February 2011, pp. 115-118.

[7] D. H. Ju, "A Concerted Effort towards Flourishing Global Software Development," Proceedings of the 2006 International Workshop on Global Software Development for the Practitioner, Shanghai, 23 May 2006, pp. 62-65. doi:10.1145/1138506.1138521

[8] V. Casey, "Imparting the Importance of Culture to Global Software Development,” ACM Inroads, Vol. 1, No. 3, 2010, pp. 51-57.

[9] P. T. Nguyen, M. A. Babar and J. M. Verner, "Critical 
Factors in Establishing and Maintaining Trust in Software Outsourcing Relationships," Proceedings of the 28th International Conference on Software Engineering, Shanghai, 20-28 May 2006, pp. 624-627.

[10] G. O. Wiredu, "A Framework for the Analysis of Coordination in Global Software Development," Proceedings of the 2006 International Workshop on Global Software Development for the Practitioner, Shanghai, 23 May 2006 , pp. 38-44. doi:10.1145/1138506.1138516

[11] E. Carmel and P. Abbott, "Configurations of Global Software Development: Offshore versus Nearshore," Proceedings of the 2006 International Workshop on Global Software Development for the Practitioner, Shanghai, 23 May 2006, pp. 3-7.

[12] S. Ramingwong and L. Ramingwong, "The Paradoxical Relationships of Risks and Benefits in Offshore Outsourcing of Software Projects,” The Open Software Engi- neering Journal, 2009, Vol. 3, pp. 35-38.

[13] O. Hazzan and Y. Dubinsky, "Can Diversity in Global Software Development Be Enhanced by Agile Software Development?” Proceedings of the 2006 International Workshop on Global Software Development for the Practitioner, Shanghai, 23 May 2006, pp. 58-61. doi:10.1145/1138506.1138520

[14] E. Ó Conchúir, P. J. Ågerfalk, H. H. Olsson and B. Fitzgerald, "Global Software Development: Where Are the Benefits?” Communications of the ACM, Vol. 52 No. 8, 2009, pp. 127-131.

[15] S.-O. Setamanit, W. Wakeland and D. Raffo, "Planning and Improving Global Software Development Process Using Simulation,” Proceedings of the 2006 International Workshop on Global Software Development for the Practitioner, Shanghai, 23 May 2006, pp. 8-14. 